\title{
The role of attention in visual processing
}

\author{
John H. R. Maunsell* and Erik P. Cook \\ Howard Hughes Medical Institute and Division of Neuroscience, Baylor College of Medicine, One Baylor Plaza, S-603, \\ Houston, TX 77030, USA
}

\begin{abstract}
Attention to a visual stimulus typically increases the responses of cortical neurons to that stimulus. Because many studies have shown a close relationship between the performance of individual neurons and behavioural performance of animal subjects, it is important to consider how attention affects this relationship. Measurements of behavioural and neuronal performance taken from rhesus monkeys while they performed a motion detection task with two attentional states show that attention alters the relationship between behaviour and neuronal response. Notably, attention affects the relationship differently in different cortical visual areas. This indicates that a close relationship between neuronal and behavioural performance on a given task persists over changes in attentional state only within limited regions of visual cortex.
\end{abstract}

Keywords: attention; visual cortex; single unit; macaque monkey; performance

\section{INTRODUCTION}

Attention is an important factor in sensory processing. Directing attention to a particular location in the visual field improves detection and discrimination, and shortens reaction times in that location relative to others (see Pashler 1998). When behavioural performance is near threshold, attention can reliably make the difference between success and failure. Attention also affects the responses of sensory neurons. Neurons typically respond more strongly when the stimulus that drives them is the focus of attention (see Braun et al. 2001). Behavioural performance depends on the activity of sensory neurons. Little is known, however, about how the behavioural effects of attention are related to the changes that it produces in sensory representations.

This question can be approached experimentally because there appears to be a close relationship between behavioural performance on a particular task and the activity of individual sensory neurons. When the sensitivity of individual neurons is compared with the behavioural capabilities of the organism, the ability of a neuron to report about a stimulus can be about as good as that of the observer, provided the task involves stimuli that are closely matched to the preferences of the neuron in question (reviewed by Parker \& Newsome 1998). This close correspondence between neuronal and behavioural performance indicates that behavioural performance depends on, and closely follows, the selectivity of sensory neurons (Barlow 1985).

Given this correspondence between neuronal and behavioural performance, and the observation that attention changes the responses of sensory neurons, it is natural to ask whether effects of attention on behavioural perform-

*Author for correspondence (maunsell@bcm.tmc.edu).

One contribution of 14 to a Discussion Meeting Issue 'The physiology of cognitive processes'. ance might be fully explained by changes that attention causes in the responses of sensory neurons. To address this issue, it is necessary to consider the magnitude and quality of effects that attention has on neuronal responses. In the sections that follow, we consider attentional effects on neuronal responses in the context of visual cortex, for which the most detailed and extensive data are available. We then describe recent experiments from our laboratory that directly examine how attention can affect the relationship between neuronal and behavioural performance.

\section{THE MAGNITUDE OF MODULATION OF NEURONAL RESPONSES BY ATTENTION}

Spatial attention has been found to affect neuronal responses in every visual cortical area examined. Most neurons respond more strongly when the subject attends to a stimulus within their receptive field, compared with attention to another stimulus far from the receptive field. However, the amount by which attention alters neuronal responses can vary greatly between different visual areas, and between neurons within a given visual area. Task demands can also affect the amount by which attention alters responses.

Within any visual area, neurons differ in the degree to which they are influenced by spatial attention. Figure 1 shows a representative distribution of attentional modulations, based on a sample of neurons from area V4 in monkey visual cortex. The average modulation was a $26 \%$ increase in response when attention was directed to the receptive field stimulus, but the responses of some neurons were twofold or threefold stronger. The responses of other neurons were not obviously affected by attention, and still others responded more strongly when attention was directed away from the receptive field. A broad range of effects that includes both positive and negative modulations is typical for studies of attention in visual cortex. Why different neurons show different degrees of attentional modulation is not understood, nor has the degree 


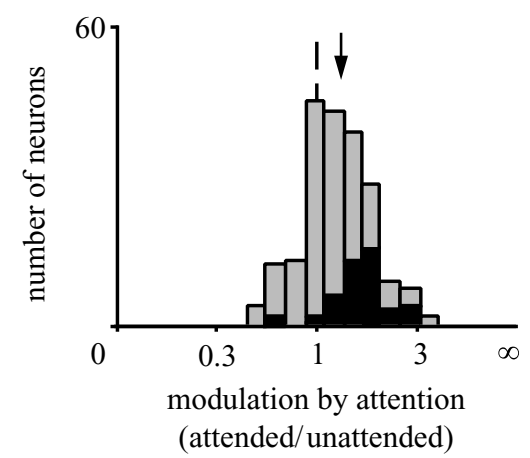

Figure 1. Typical distribution of attentional modulation for visual cortex. Responses were recorded from 197 neurons in area V4 while monkeys performed a task that directed their attention towards or away from a stimulus in the receptive field of the neuron being recorded. Most neurons responded more strongly when the animal paid attention to the stimulus in the receptive field (average increase $26 \%$, indicated by arrow). However, the distribution was broad, and some neurons responded more strongly when the animal directed its attention away from the receptive field. Response changes that were statistically significant are shown in black. (Data from McAdams \& Maunsell (1999a).)

of attentional modulation been shown to correlate with other properties of the neurons.

Attentional modulation also differs between visual areas. Modulation by attention is typically weakest in the earliest stages of visual cortex, and strongest in the latest stages. This increase has not been studied extensively, but it is apparent in comparing results from different reports. The best data come from individual studies that have recorded from different cortical areas in the same animals while they performed a given task. Figure 2 shows the average attentional modulation in different cortical areas that were examined in this way in our laboratory. Average attentional modulation is plotted as a function of the level of cortical processing, as defined by the hierarchy of Felleman \& Van Essen (1991). In each study, areas at later stages had stronger average attentional modulation. These results were based on extracellular recording of individual action potentials, but stronger modulation at later stages of visual cortex has also been seen by using current source density measurements (Mehta et al. 2000).

It is not known why attentional modulations are stronger in later cortical levels. While it is tempting to imagine that neuronal activity related to attention accumulates in successive levels of processing, this seems unlikely given that sensory responses arriving from different sources do not appear to accumulate in this way. Similarly, the notion that neuronal signals related to attention are inserted at the latest stages of processing and diminish as they are fed back to earlier stages seems unlikely. It seems probable that the degree of modulation is optimized for each cortical level. There is little reason to believe that the cerebral cortex would be unable to prevent undesirable accumulation or diminution of signals that altered sensory responses.

In addition to the range of attentional modulation seen within and between visual areas, individual neurons can show different degrees of attentional modulation depending on task demands. Task demand can be affected either by the number of relevant items or by the com-

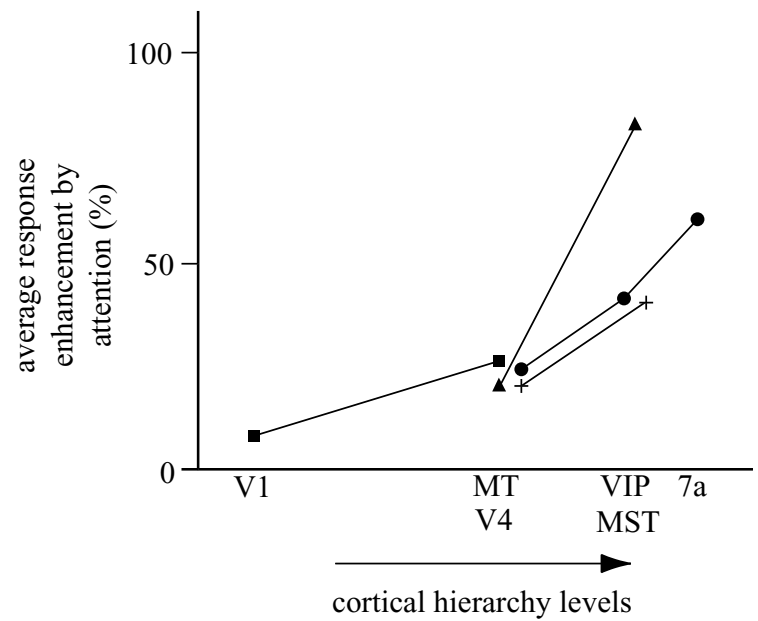

Figure 2. Average attentional modulation in visual cortical areas. The average response enhancement reported from studies that measured the effects of attention in two or more cortical areas in the same subjects while they performed a given task are shown. Positions on the $x$-axis are assigned according to the hierarchical levels defined by Felleman \& Van Essen (1991). More attention modulation is found in later stages of cortical processing (squares, McAdams \& Maunsell (1999a); crosses, Treue \& Maunsell (1999); circles, Ferrera et al. (1994); triangles, Cook \& Maunsell (2002)). (Based on Cook \& Maunsell (2002).)

plexity of the processing to be performed on the relevant items (Lavie \& Tsal 1994; Urbach \& Spitzer 1995; Sade \& Spitzer 1998). Mountcastle et al. (1981, 1987) showed that most neurons in area $7 \mathrm{a}$ and neighbouring regions were far more responsive when an animal was engaged in a visual task than when the same retinal stimuli were presented during periods of alert wakefulness when no fixation was required. Neuronal responses in inferotemporal cortex have also been found to be progressively stronger as a fixating animal goes from a situation where the stimulus is irrelevant, to monitoring the stimulus to detect its dimming, to discriminating the shape or texture of the stimulus (Spitzer \& Richmond 1991). Similarly, the responses of V4 neurons are stronger when an animal performs a more difficult version of an orientation change detection task (Spitzer et al. 1988). The effects of task demand may be related to arousal or vigilance, but they can affect spatial attention. In some circumstances, modulation by spatial attention can be more than twice as strong during a difficult task (Boudreau \& Maunsell 2001).

The attentional modulation of the responses of individual neurons can vary not only between tasks but also within trials. Motter (1994) provided a clear example of the dynamics of attention. He trained monkeys to do a task in which an instruction redirected their spatial attention in the middle of some trials. Neurons in V4 changed their responses within a few hundred milliseconds of the new instruction. Other studies have shown that attentional modulation can vary systematically during the course of task trials (e.g. McAdams \& Maunsell 1999a; Reynolds et al. 2000). These observations indicate that attentional modulation of individual neurons is dynamic, and varies over a time-course of no more than a few hundred milliseconds.

In summary, attentional modulation of neuronal signals 
varies substantially within and between areas, and the modulation of individual neurons can vary depending on task demands, and probably other factors. This variable aspect of attentional modulations makes comparisons between different experiments problematic. It also means that there is little point in seeking a specific, fundamental value to describe the effect of attention on a neuron or area.

Although attention substantially alters sensory responses (e.g. figures 1 and 2), it does not usually eliminate responses to unattended stimuli. Occasional neurons are described that respond only when the animal attends to the stimulus in their receptive field, but no report has described this as typical for neurons in a visual area. While it is likely that more neurons would show this sort of gating by attention in extremely demanding tasks, those tasks are not representative of the effort of most visual tasks. When animals perform tasks that approximate the attentional demands of everyday visual functions, attention substantially emphasizes the cortical representation of behaviourally relevant stimuli relative to others, but does not obliterate most signals about other stimuli.

\section{THE QUALITY OF MODULATIONS OF NEURONAL RESPONSES BY ATTENTION}

In addition to changing the magnitude of neuronal responses, attention might affect the information contained in the neuronal signal. For example, does attention change the preferred orientation or speed, or the sharpness of tuning for different stimulus attributes? The relationship between tuning for stimulus dimensions and the quality of information conveyed by a population of neurons is complex (Zhang \& Sejnowski 1999), and it is difficult to argue that attention should have a particular effect on selectivity to produce better behavioural performance. Nevertheless, it is important to examine whether changes in neuronal stimulus selectivity are associated with attention. Only a handful of studies have addressed this question, but they suggest that attention does not systematically alter the selectivity of neurons. Instead, attention appears to act by increasing the responsiveness of neurons without changing the selectivity or reliability of responses.

McAdams \& Maunsell (1999a) measured the orientation tuning of V4 neurons when attention was directed towards the orientation of a stimulus in their receptive fields, and when it was directed to the colour of a distant stimulus. Responses were stronger with attention directed to the receptive field, but responses to all orientations were increased by the same proportion. Figure 3 shows average orientation tuning curves for the two conditions in that study. Although responses were on average ca. $30 \%$ stronger when attention was directed to the receptive field, there was no systematic change in the sharpness of orientation tuning. Treue \& Martínez Trujillo (1999) similarly found that attention increased the strength of responses of neurons in MT but did not change the sharpness of their directional tuning. In V4, the spatial profiles of receptive fields appear to scale proportionally when attention is directed at different locations near the receptive field, without changing the shape of the receptive field profile (Connor et al. 1996, 1997). Additionally, orientation tuning curves measured in inferotemporal cortex

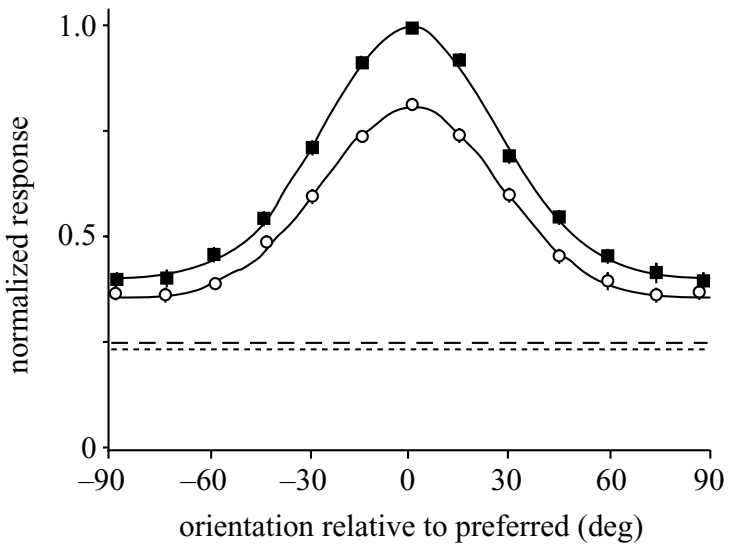

Figure 3. Average normalized tuning curves for 262 V4 neurons tested when the stimulus was attended (filled symbols) or ignored (open symbols). Tuning curves for each cell were normalized to the peak response in the attended condition, and shifted left or right to bring each cell's preferred orientation into alignment. Gaussian functions were then fitted to the averaged data for each behavioural state. Dashed and dotted lines represent the average spontaneous activities in the attended and unattended states respectively. Although the amplitude of the normalized tuning curves changed markedly when the animal attended to the stimulus (amplitude: 0.46-0.60), the width did not $\left(\sigma=37.8-37.5^{\circ}\right.$. (Data from McAdams \& Maunsell (1999a).)

using reward-contingent and non-reward-contingent stimuli differ in response strength but not in the sharpness of tuning (Vogels \& Orban 1994).

Similarly, attention does not appear to alter the underlying reliability of cortical responses. When the same stimulus is presented many times, cortical neurons respond with different numbers of spikes for different presentations. This variability is captured by the relationship between the mean strength of a neuron's response and the variance of the response measured over many presentations of the same stimulus. In most cases, the variance of the spike counts is proportional, and generally close to, the mean spike count (e.g. Tolhurst et al. 1981; Geisler \& Albrecht 1997). Attention does not affect the relationship between the mean and variance of responses for neurons in area V4 (McAdams \& Maunsell 1999b). Although attention changes the average responsiveness of neurons, it does not alter the variance associated with a given level of response.

While in many situations attention appears to increase the sensitivity of neurons without obviously affecting their underlying stimulus selectivity, there are cases where attention does alter selectivity. For example, Moran \& Desimone (1985) described how attention altered the receptive fields of V4 neurons to emphasize the region around the stimulus to which the subject was attending (see also Luck et al. 1997). A shift in a neuron's receptive field profile is not consistent with a simple change in its sensitivity. Nevertheless, a shift in receptive fields can be explained by simple changes in sensitivity that occur at earlier levels in cortical processing (see Maunsell \& McAdams 2001), and are consistent with a mechanism that increases responses to attended stimuli relative to others in the scene.

While more stimulus dimensions and more visual areas 
must be examined before firm conclusions can be drawn, the results described above indicate that attention selectively enhances responses to attended stimuli, without changing the feature selectivity of the responsive neurons. This enhancement is similar to what occurs when neurons are presented with stimuli that are more intense, or better suited to the stimulus preferences of the neuron, such as better matched to a preferred colour or speed of motion. For example, increasing stimulus contrast typically makes a neuron's response stronger without changing its selectivity for orientation, direction, spatial frequency or stimulus position (Dean 1981; Holub \& Morton-Gibson 1981; Sclar \& Freeman 1982; Albrecht \& Hamilton 1982; Skottun et al. 1987; Geisler \& Albrecht 1997). Similarly, selectivity for one stimulus dimension generally does not differ greatly between measurements made using a stimulus that is optimal or suboptimal for another stimulus dimension (see McAdams \& Maunsell 1999a); e.g. measurements of direction selectivity at an optimal and suboptimal speed, (Rodman \& Albright 1987), although there are exceptions (e.g. Roy \& Wurtz 1990). Attention also mimics the way that stimulus changes alter responses without affecting the relationship between response strength and response variance (Dean 1981; Tolhurst et al. 1983; Snowden et al. 1992).

The notion that attention acts in a manner similar to increasing stimulus intensity is supported by the recent results of Reynolds et al. (2000). They measured responses of V4 neurons to stimuli of different contrasts, which were or were not attended by the subject. Attention increased neuronal responses in a manner that was similar to the effect of increasing the contrast of each stimulus by a given amount.

\section{ATTENTION, NEURONAL RESPONSES AND BEHAVIOURAL PERFORMANCE}

Attention does not appear to alter the nature or structure of the representations in cortex. Instead it serves to highlight particular stimuli within the existing representational framework. This observation suggests an explanation for the behavioural effects of selective visual attention. Focusing attention on a stimulus influences neuronal responses in a way that is similar to increasing stimulus intensity. Like attention, increased stimulus intensity results in neurons responding more strongly, but does not sharpen their tuning for different stimulus features. If the effect of attention on neurons was functionally equivalent to higher intensity or salience of the attended stimuli, the behavioural advantages associated with selective attention-better detection, discrimination and reaction time-might be explained by changes in the representations in sensory cerebral cortex. Thus, attention may make attended stimuli appear more intense or salient by enhancing their representations in sensory cortex.

The possibility that changes in neuronal responses in sensory cortex can fully account for the behavioural advantages associated with attention raises an important question about the relationship between neuronal sensitivity and behavioural capabilities. As mentioned in $\ 1$, studies that have compared neuronal and behavioural performance have often found that under appropriate conditions, the signals of individual neurons can be as reliable as the subject's behaviour in distinguishing between stim- uli (see Parker \& Newsome 1998). Does the correspondence between neuronal and behavioural performance remain fixed when attention alters neuronal responses? We examined this question by comparing neuronal and behavioural responses between different attentional states (Cook \& Maunsell 2002).

Because many studies of neuronal performance in visual cortex have been done in areas with directionally selective neurons, we trained two monkeys (Macaca mulatta) to do a motion detection task that made it possible to measure neuronal and behavioural responses to stimuli in conditions of high and low attention. The animal viewed a screen on which two patches of dynamic random dots appeared. At the start of each trial, there was no net motion in either patch of dots ( $0 \%$ motion coherence), and the animal's task was to rapidly release a lever when dots of either patch began to move coherently in one direction. The motion signal on each trial was randomly selected from preset levels of coherence. One of the patches filled the receptive field of the neuron being recorded, and coherent motion in either patch matched the preferred direction and speed of the neuron.

Motion appeared in only one patch on each trial, and the animal was cued at the start of the trial as to which patch was likely to contain the motion. Critically, the cue was valid on only about $80 \%$ of the trials. On the remaining trials motion occurred at an uncued location. These invalidly cued trials made it possible to measure behavioural and neuronal responses to stimuli to which the animal directed relatively little attention (Posner 1980). The behavioural performance of both animals showed that they used the cue on each trial to direct most of their attention to the patch of dots that was likely to contain the motion. Moderate motion coherence was typically detected on more than $80 \%$ of trials when it appeared in the cued location, but on fewer than $40 \%$ of trials when it appeared in the uncued location. (These behavioural data cannot distinguish whether the animal simultaneously attended to the cued and uncued locations with different amounts of attention allocated to each, or if instead all of its attention was assigned to one patch at any instant, with the proportion of time spent attending to each patch depending on the cue. However, the neurophysiological recordings indicate that the animal allocated different amounts of attention to the two patches, and that these amounts did not vary during the stimulus presentations (see Cook \& Maunsell 2002, figure 10).)

We recorded from neurons in MT and VIP. We chose these areas because both contain neurons that respond selectively to motion. Responses from a typical MT neuron are shown in figure 4 . The filled symbols in figure $4 a$ show the average response of the neuron to different levels of motion coherence in the receptive field when the animal had been cued to attend to that location. The dashed line shows the average level of response to the $0 \%$ motion stimulus that preceded the motion. As expected, stronger motion produced stronger responses. The open symbol shows the neuron's average response to the medium motion strength (15\% coherence) in the receptive field on trials in which the animal had been instructed to attend to the other location. Although the same motion appeared in the receptive field, the response was weaker. While measurements of responses to unattended stimuli of dif- 


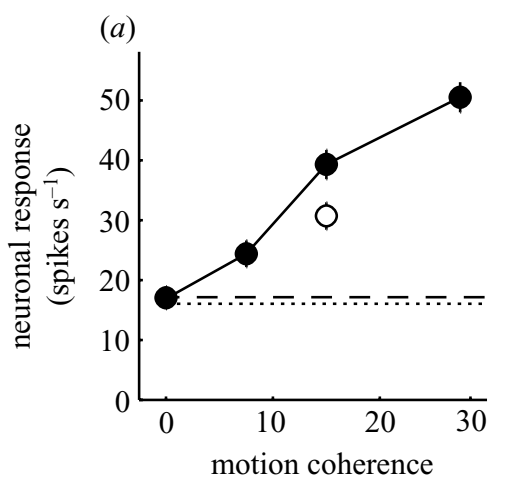

(c)

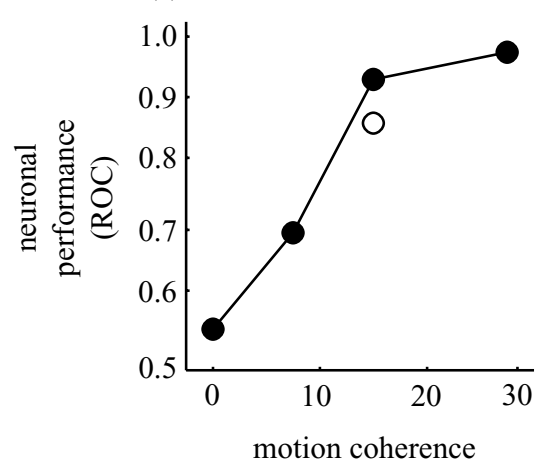

(b)

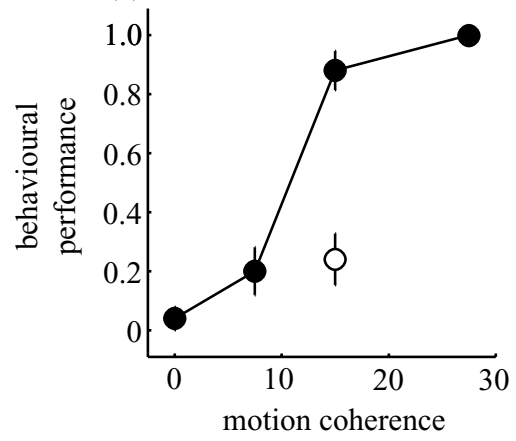

$(d)$

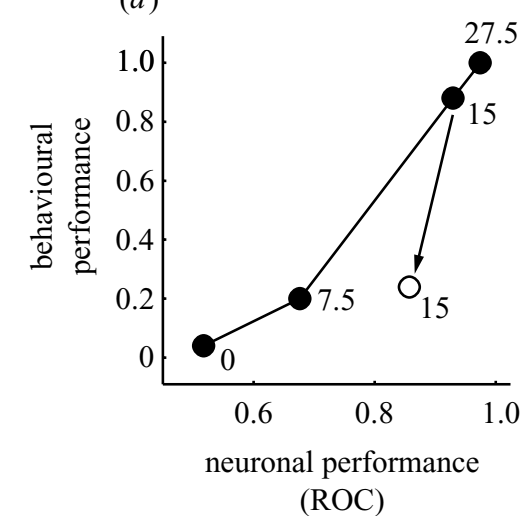

Figure 4. Responses of a representative MT neuron. (a) Responses to motion stimuli with different levels of coherence. Motion coherence corresponds to the strength of the motion signal. At $0 \%$ coherence, there is no net motion. Each filled circle is the average response during the first $300 \mathrm{~ms}$ after the onset of motion with the coherence indicated. The dashed line is the average response to the $0 \%$ coherence stimulus that appeared before the onset of coherent motion. The open circle is the response to $15 \%$ coherent motion in the receptive field that appeared on trials when the animal's attention had been directed away from the receptive field. The dotted line is the response to $0 \%$ coherent motion in those trials. Error bars in all panels are the standard errors of the means, and are sometimes smaller than the symbols. As expected, directing attention away from the receptive field reduced neuronal responses. (b) Behavioural performance for the same trials used to collect the neuronal responses. The open circle shows that the animal's ability to detect the $15 \%$ coherent stimulus was greatly reduced when its attention was directed away from the location of the motion. (c) Neuronal performance. Neuronal responses were converted into performance using ROC based on $300 \mathrm{~ms}$ immediately before and after the onset of motion. (d) The data from $(b$ and $c$ ) are replotted parameterized by motion coherence. The value by each symbol gives the associated motion coherence. The data from the cases when the animal attended to the location of the motion define a function, but the open symbol, from trials on which the animal's attention was directed away from the stimulus, does not lie on that function. Thus, directing attention away from the stimulus does not have the same effect as reducing motion coherence while keeping attention fixed.

ferent motion coherences would have been valuable, it was not practical to collect those data because invalid cues had to be restricted to a small percentage of the trials.

Figure $4 b$ shows the animal's behavioural performance on the same trials on which the neuronal responses were recorded. The filled symbols plot performance when the animal was attending to the receptive field and the motion occurred there. The open symbol is the behavioural performance for detecting the onset of the medium motion coherence in the receptive field when attention had been directed to the other patch of random dots. Giving the animal an invalid cue caused performance to drop from ca. $85 \%$ correct to only ca. $25 \%$ correct.

The question we wanted to address was whether the decreases in neuronal and behavioural performance caused by directing attention were the same as those that might have occurred by keeping attention fixed and reducing the motion strength: is behavioural performance the same for an attended, weak stimulus and an unattended, strong stimulus if they both produce the same neuronal response? To examine this, we converted the neuronal responses into a measure of performance using ROC analysis (Green \& Swets 1966). This analysis evaluates how well an ideal observer could detect the presence of motion if the only signal available were the spikes of the neuron. For this analysis, we counted spikes in $300 \mathrm{~ms}$ windows immediately before and after the onset of the motion, for each of the conditions. The ROC performance based on the neuronal signal is plotted in figure $4 c$ as a function of motion coherence. ROC values run from 1.0, which corresponds to perfect performance, to 0.5 , which corresponds to performance that is no better than guessing. The neuronal response to the medium motion coherence could support good performance when the receptive field location had been cued, and the neuronal performance was only slightly reduced when the same stimulus appeared with attention directed elsewhere (open symbol). 
(a)

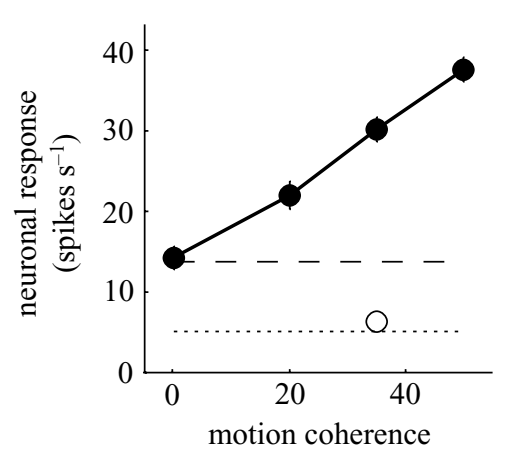

(c)

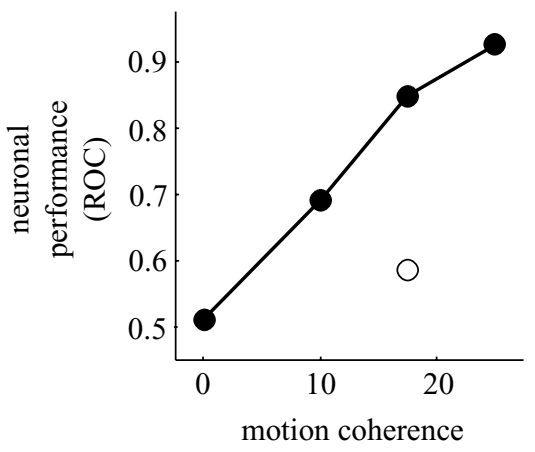

(b)

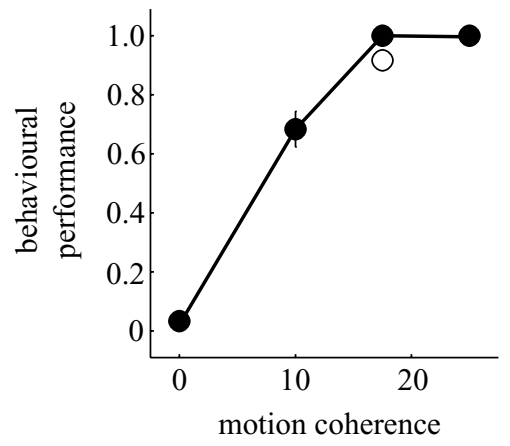

(d)

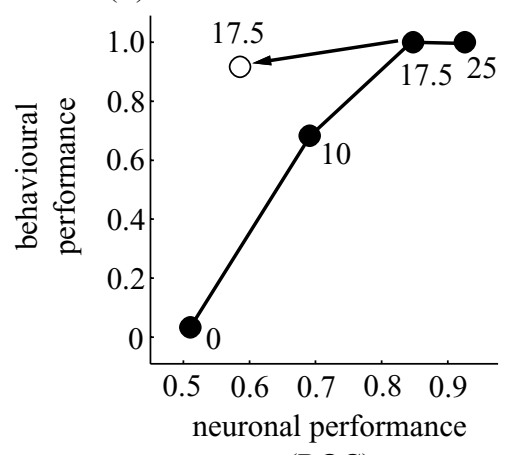

(ROC)

Figure 5. Responses of a representative VIP neuron. Same format as figure 4. (a) Responses to motion stimuli with different levels of coherence; $(b)$ behavioural performance for the same trials used to collect the neuronal responses; $(c)$ neuronal performance; and $(d)$ the data from $(b)$ and $(c)$ replotted parameterized by motion coherence. Attention modulated the neuronal response far more than expected based on its modulation of behavioural performance.

One way to assess whether shifting attention is equivalent to changing stimulus strength is to plot neuronal and behavioural performance against one another, parameterized by the coherence of the motion. In figure $4 d$, each point represents the behavioural and neuronal performance for a particular motion coherence, as indicated by the numbers beside each point. If the effect of attention were equivalent to changing stimulus strength, then the point for the invalid cue should lie on the function described by the filled symbols. In this case, it does not: it lies substantially to the right of the function. Shifting attention modulated the neuronal response by less than would be needed to account for the modulation of behavioural performance. This result was typical for neurons in MT. The responses of most MT neurons were weakly affected by attention. The average modulation among 93 neurons was about a $15 \%$ change in response. Attention had a more pronounced effect on behavioural performance, and for most neurons the attentional modulation of the neuronal response was too small to account for the attentional modulation of the behavioural response.

A different result was found in VIP. VIP receives input from MT (Maunsell \& Van Essen 1983; Ungerleider \& Desimone 1986), and like MT, most of its neurons are direction selective (Colby et al. 1993; Cook \& Maunsell 2002). Most neurons in VIP had strong attentional modulation; too strong to match the behavioural modulation by attention. The responses of a representative VIP neuron are shown in figure 5, which has the same format as figure
4. Figure $5 a$ shows that the neuron's responses were profoundly affected by attention. Although the neuron responded strongly to the medium motion coherence when the animal was attending to the receptive field, the same stimulus produced almost no response when the animal was attending elsewhere. The dashed and dotted lines show that attention also affected the response to the $0 \%$ motion. The response to unattended moderate motion was less than the response to the $0 \%$ coherent motion when it was attended. Although the neuron's responses were devastated when attention was directed elsewhere, in this particular case the animal's behaviour was only slightly reduced (figure $5 b$ ). Plotting neuronal performance against behavioural performance showed that attention did not have the same effect as changes in the motion coherence of attended stimuli (figure $5 d$ ). Unlike MT, however, the point for the unattended stimulus lies to the left of the function defined by the points for the attended stimuli. This means that the attentional modulation of the neuronal response was stronger than what would be expected based on the modulation of the behavioural response. The responses of most neurons in VIP were strongly modulated by attention, and it was common to find that the neuronal modulation was greater than the associated behavioural modulation.

The responses of all the neurons recorded in MT and VIP are summarized in figure 6. Data from each animal and each area are plotted separately. Each plot shows the average neuronal and behavioural performance associated 
(a)

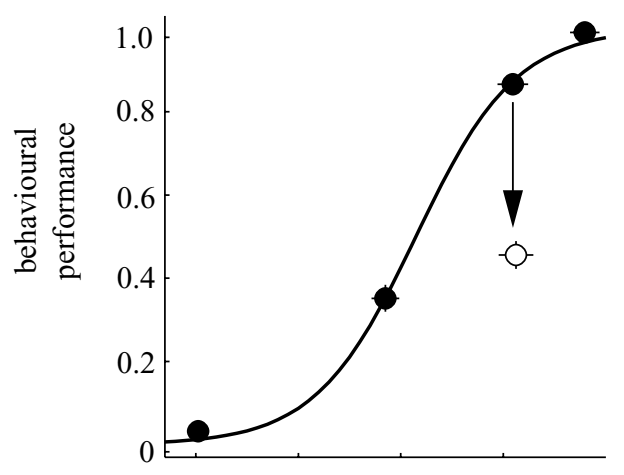

(c)

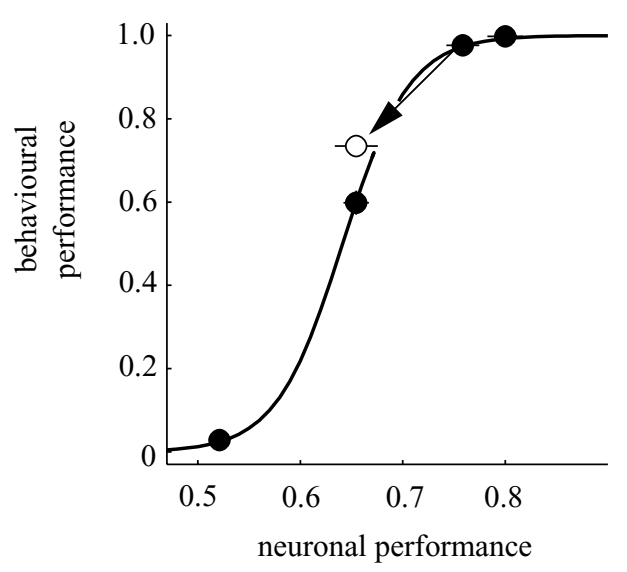

(ROC) (b)

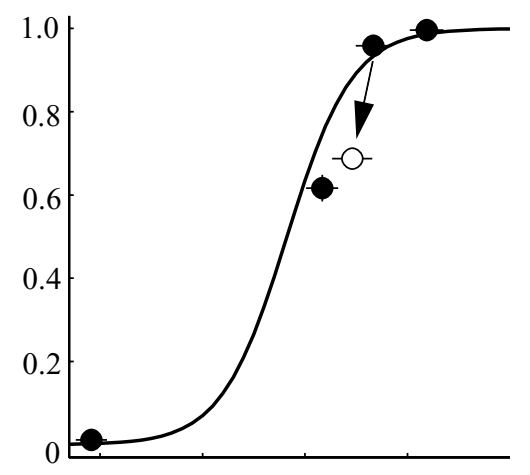

(d)

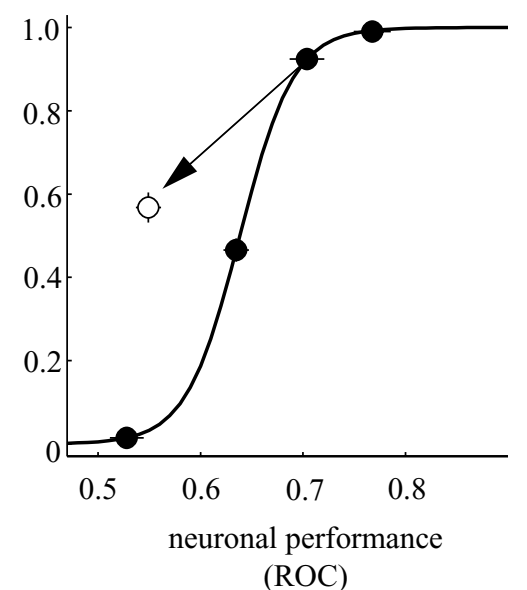

Figure 6. Summary of the effects of attention on neuronal and behavioural performance. (a) Animal 1, MT; (b) animal 2, MT; (c) animal 1, VIP; and (d) animal 2, VIP. Each panel is a plot of neuronal versus behavioural performance in the form shown in figures $4 d$ and $5 d$. The filled circles are the average performance for high, medium and low motion coherence and solid bars are sigmoidal fits. Averages from individual neurons were combined to produce these plots. Error bars are the standard errors of the means. Open circles are performance for the medium coherence when the subject's attention was directed away from the stimulus. Although the subjects differed somewhat, the unattended points for the MT are both to the right of the functions for the attended stimuli, and the unattended points for the VIP are both to the left of the functions for the attended stimuli. The effects of attention are not readily equated to changes in motion coherence.

with high, medium and low levels of motion coherence, and for the medium motion coherence when it was in the uncued location. For MT data from both animals, the means for the performance on the uncued condition lie to the right of the function defined by the cued condition, although by different amounts for the two animals. For VIP, the means for the performance on the uncued condition lie to the left of the function, although barely so for animal 1. The differences between the two animals arise in part from greater overall attentional modulation for animal 2. The average neuronal modulation for animal 2 was $28 \%$ for MT and $94 \%$ for VIP, compared with $12 \%$ for MT and $68 \%$ for VIP in animal 1 .

The analyses in figures 4-6 compare neuronal performance measured using ROC against behavioural performance measured as a percentage of stimuli correctly detected. Might different measures reveal a better correspondence between neuronal and behavioural, or better correspondence between the animals? For example, neuronal response might be measured as absolute or relative rate of firing, and behavioural response might be measured as reaction time. We have tested these and other measures of neuronal and behavioural performance. Different analyses change the shapes of the functions and shift the unattended points slightly left or right, but none changed the qualitative appearance of the results shown in figure 6 (see Cook \& Maunsell 2002). We do not believe that any analysis could bring all the unattended points onto the functions defined by the attended points.

These results show that the relationship between neuronal response and behavioural performance can be changed by attention. Although attention substantially affected behavioural performance, it had relatively little effect on the responsiveness of MT neurons. Thus, similar levels of neuronal performance in MT were associated with different levels of behavioural performance. In VIP, the relationship between neuronal response and performance was also affected by attention, but in this case the attention affected neuronal performance more than behavioural performance.

While there are several possible reasons for this outcome, differences in the amount of attentional modulation across visual cortex provide a probable explanation. Attentional modulation of neuronal responses is greater in later stages of cortical processing (figure 2). Although the origin of this difference is not known, it should have consequences for the relationship between neuronal and behavioural performance. If stimulus-response functions are 
(a)

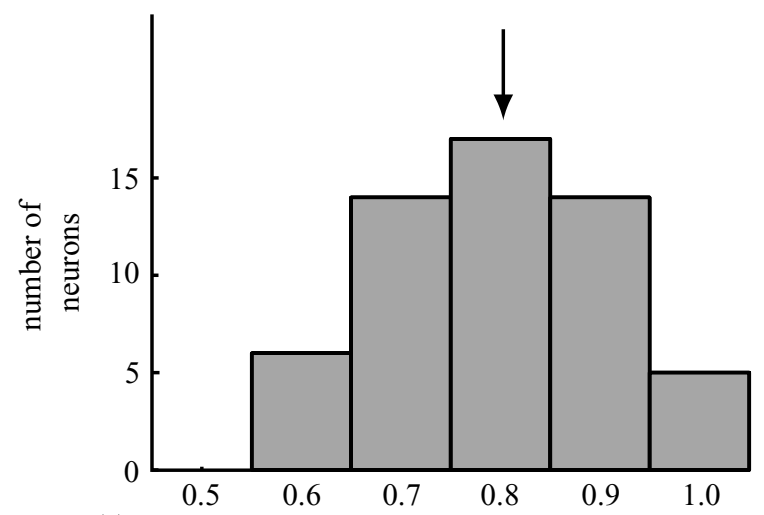

(c)

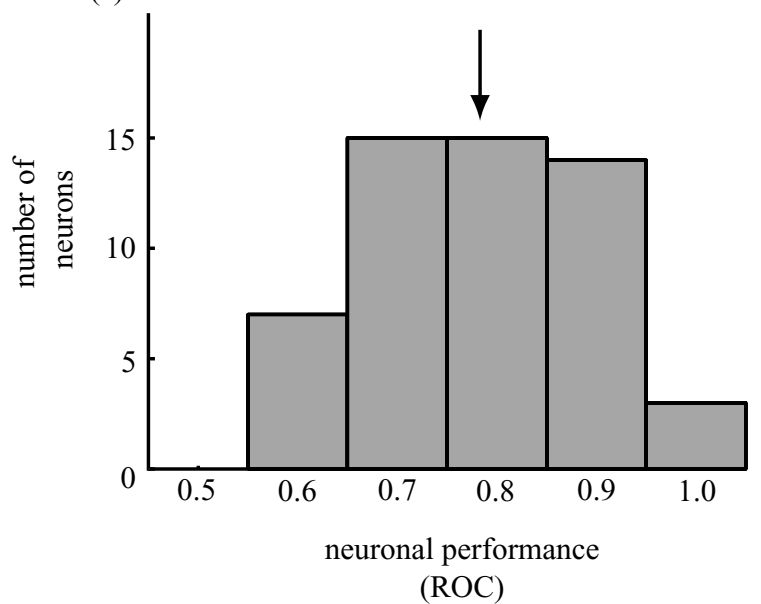

(b)

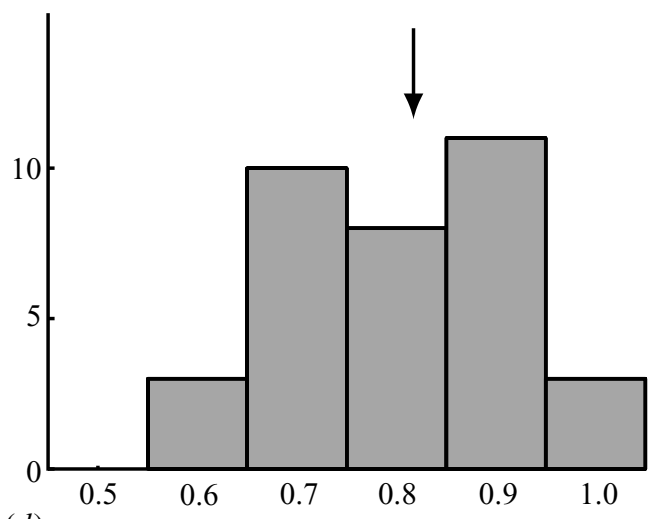

(d)

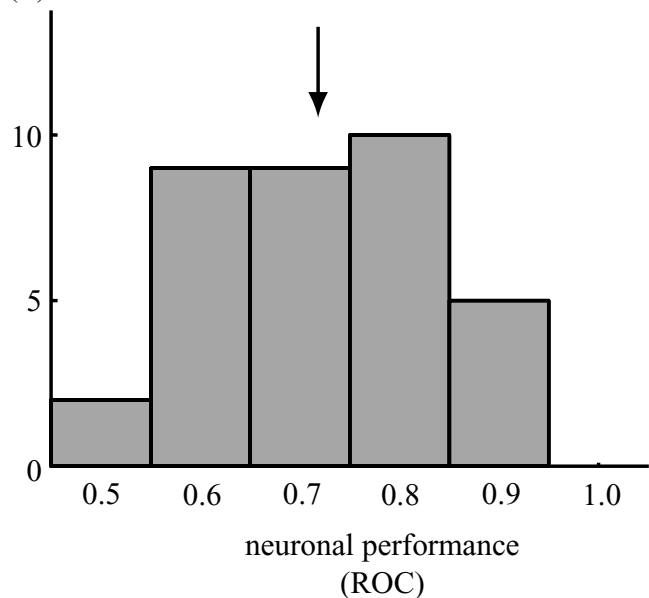

Figure 7. Neuronal performance detecting the onset of stimulus motion. (a) Animal 1, MT; (b) animal 2, MT; (c) animal 1, VIP; and $(d)$ animal 2, VIP. ROC analysis was carried out using 300 ms periods immediately before and after the onset of medium motion coherence when it occurred in the cued location. The median values for the MT were 0.80 and 0.82 for animals 1 and 2, and the median values for the VIP were 0.78 and 0.72 respectively. Thus, the performance of MT neurons was slightly superior. The MT performance was also better for low and high motion coherence.

similar between areas (as they are for MT and VIP; figures $4 a$ and $5 a$ ), then a given change in behavioural performance caused by changes in attention will be matched only by a specific change in neuronal response. Because attentional modulation varies between areas, a match between the change in neuronal response and behaviour can be centred only on a particular level of cortical processing. It is possible that the neurons at some level between MT and VIP had an average attentional modulation that was well matched to the average behavioural modulation. If so, then neurons in this region might have responses that closely matched behavioural performance across manipulations of either stimulus strength or attentional state.

The notion that behavioural performance might be most closely associated with neurons that are intermediate in cortical processing raises the question of why behaviours would not be based on the neurons with the greatest attentional modulation. The answer is probably that the neurons with the greatest attentional modulation do not have response properties that are optimal for the task. Neurons with the greatest attentional modulation typically lie at the highest levels of cortical processing, and have complex response properties. For example, although neurons in both MT and VIP are directionally selective, VIP neurons have more elaborate response properties. VIP neurons respond to several types of visual and extraretinal signals, including optic flow patterns, tactile stimulation of the face, vestibular stimulation, and the position of the eyes in the orbits (Schaafsma \& Duysens 1996; Schaafsma et al. 1997; Colby \& Goldberg 1999). Although these neurons are strongly modulated by attention, their specialization for these more complex response properties is likely to make them less reliable than other neurons for detecting the onset of the motion used in the experiments described here.

An analysis of the responses we collected from MT and VIP indicates that neurons in VIP are not better suited than MT neurons for detecting the motion used in our task. Figure 7 shows distributions of neuronal performance for the two areas. A broad range of performance was found in both areas, but in both animals the median performance was slightly better for MT. These distributions are based on responses to the medium motion coherence when it appeared in the cued location, but MT performance was also better for the other levels of motion coherence. Thus, neurons at later levels of cortical processing are not necessarily better suited for all tasks.

We know of no data that strongly suggest that behavioural performance should always depend on neurons in the highest levels of visual cortex. Basing performance on those representations would introduce a substantial restriction, because far fewer neurons would be available 
if only the highest levels are accessed. Rather than depending on those neurons that are most modulated by attention, behavioural performance is likely to be based on those neurons with response properties that provide the most sensitive signals for the current task. This suggestion is consistent with observations on perceptual learning that indicate that earlier or later cortical levels are involved in learning different types of visual discriminations (Ahissar \& Hochstein 1997). In the case of our motion detection task, neurons with the greatest sensitivity might be found at a level intermediate to MT and VIP, and behaviours might be most closely linked to the performance of those neurons. Were that the case, it would be natural for behaviour to be modulated by attention to the same extent that those best-suited neurons were modulated by attention. If, in a different task, behavioural performance was best served by representations in later stages of visual cortex, where attentional modulation is strong, we would expect that behaviour would be more susceptible to attentional modulation.

\section{CONCLUSION}

More data will be needed before we will know whether the relationship between neuronal and behavioural performance persists across attentional states for any region of the visual cortex. Nevertheless, the results described here indicate that a correspondence across attention states will be limited to a restricted level of processing, the location of which will vary depending on the task being performed.

Even if neurons at a given level do preserve a fixed relationship between neuronal and behavioural performance across attentional states for a task, it would not necessarily mean that those neurons were more or less important than others for performing the task. Similarly, it would say nothing about how large a pool of neurons contribute to a decision. A cortical region that had a close match would be a good candidate for being the centre of the relevant neurons, but the pool size might be large or small without appreciably affecting the mean neuronal performance. Additionally, a correspondence between average neuronal performance and average behavioural performance would not reveal which cortical region was most involved, because behavioural performance might be based on the best neurons in a population, rather than the average (see Parker \& Newsome 1998).

Much remains to be learned about how the activity of individual neurons is translated into behavioural decisions. Nevertheless, progress in this area is essential for understanding how the brain works. Manipulation of attention may prove to be a valuable approach to exploring how neurons contribute to particular behaviours.

We thank our many colleagues who contributed to the studies reviewed here and provided comments on the manuscript, including W. Bosking, C. E. Boudreau, J. J. DiCarlo, G. M. Ghose, C. Hocker, C. J. McAdams, D. Murray, T. Williford and T. Yang. Supported by NIH R01 EY05911. J.H.R.M. is an investigator with the Howard Hughes Medical Institute.

\section{REFERENCES}

Ahissar, M. \& Hochstein, S. 1997 Task difficulty and the specificity of perceptual learning. Nature 387, 401-406.

Albrecht, D. G. \& Hamilton, D. B. 1982 Striate cortex of monkey and cat: contrast response function. F. Neurophysiol. 48, 217-237.

Barlow, H. B. 1985 The twelfth Bartlett memorial lecture: the role of single neurons in the psychology of perception. Q. $\mathcal{F}$. Exp. Psychol. 37, 121-145.

Boudreau, C. E. \& Maunsell, J. H. R. 2001 Is spatial attention a limited resource for V4 neurons? Soc. Neurosci. Abstr. 27, 574.3.

Braun, J., Koch, C. \& Davis, J. L. 2001 Visual attention and cortical circuits. Cambridge, MA: MIT.

Colby, C. L. \& Goldberg, M. E. 1999 Space and attention in parietal cortex. A. Rev. Neurosci. 22, 319-349.

Colby, C. L., Duhamel, J. R. \& Goldberg, M. E. 1993 Ventral intraparietal area of the macaque: anatomic location and visual response properties. F. Neurophysiol. 69, 902-914.

Connor, C. E., Gallant, J. L., Preddie, D. C. \& Van Essen, D. C. 1996 Responses in area V4 depend on the spatial relationship between stimulus and attention. $\mathcal{F}$. Neurophysiol. 75, 1306-1308.

Connor, C. E., Preddie, D. C., Gallant, J. L. \& Van Essen, D. C. 1997 Spatial attention effects in macaque area V4. $\mathcal{F}$. Neurosci. 17, 3201-3214.

Cook, E. P. \& Maunsell, J. H. R. 2002 Attentional modulation of behavioral performance and neuronal responses in middle temporal and ventral intraparietal areas of macaque monkey. F. Neurosci. 22, 1994-2004.

Dean, A. F. 1981 The variability of discharge of simple cells in the cat striate cortex. Exp. Brain Res. 44, 437-440.

Felleman, D. J. \& Van Essen, D. C. 1991 Distributed hierarchical processing in the primate cerebral cortex. Cerebr. Cortex 1, 1-47.

Ferrera, V. P., Rudolph, K. K. \& Maunsell, J. H. R. 1994 Responses of neurons in the parietal and temporal visual pathways during a motion task. F. Neurosci. 14, 6171-6186.

Geisler, W. S. \& Albrecht, D. G. 1997 Visual cortex neurons in monkeys and cats: detection, discrimination and identification. Visual Neurosci. 14, 897-919.

Green, D. M. \& Swets, J. A. 1966 Signal detection theory and psychophysics. New York: Wiley.

Holub, R. A. \& Morton-Gibson, M. 1981 Response of visual cortical neurons of the cat to moving sinusoidal gratings: response-contrast functions and spatiotemporal interactions. F. Neurophysiol. 46, 1244-1259.

Lavie, N. \& Tsal, Y. 1994 Perceptual load as a major determinant of the locus of selection in visual attention. Percept. Psychophys. 56, 183-197.

Luck, S. J., Chelazzi, L., Hillyard, S. A. \& Desimone, R. 1997 Neural mechanisms of spatial selective attention in areas V1, V2 and V4 of macaque visual cortex. F. Neurophysiol. 77, $24-42$.

McAdams, C. J. \& Maunsell, J. H. R. 1999a Effects of attention on orientation-tuning functions of single neurons in macaque cortical area V4. F. Neurosci. 19, 431-441.

McAdams, C. J. \& Maunsell, J. H. R. $1999 b$ Effects of attention on the reliability of individual neurons in monkey visual cortex. Neuron 23, 765-773.

Mehta, A. D., Ulbert, I. \& Schroeder, C. E. 2000 Intermodal selective attention in monkeys. I. Distribution and timing of effects across visual areas. Cerebr. Cortex 10, 343-358.

Maunsell, J. H. R. \& McAdams, C. J. 2001 Effects of attention on the responsiveness and selectivity of individual neurons in visual cerebral cortex. In Visual attention and cortical circuits (ed. J. Braun, C. Koch \& J. L. Davis), pp. 103-119. Cambridge, MA: MIT. 
Maunsell, J. H. R. \& Van Essen, D. C. 1983 The connections of the middle temporal visual area (MT) and their relationship to a cortical hierarchy in the macaque monkey. $\mathcal{F}$. Neurosci. 3, 2563-2586.

Moran, J. \& Desimone, R. 1985 Selective attention gates visual processing in the extrastriate cortex. Science 229, 782-784.

Motter, B. C. 1994 Neural correlates of feature selective memory and pop-out in extrastriate area V4. F. Neurosci. 14, $2190-2199$

Mountcastle, V. B., Andersen, R. A. \& Motter, B. C. 1981 The influence of attentive fixation upon the excitability of the light-sensitive neurons of the posterior parietal cortex. $\mathcal{F}$. Neurosci. 1, 1218-1235.

Mountcastle, V. B., Motter, B. C., Steinmetz, M. A. \& Sestokas, A. K. 1987 Common and differential effects of attentive fixation on the excitability of parietal and prestriate (V4) cortical visual neurons in the macaque monkey. $\mathcal{F}$. Neurosci. 7, 2239-2255.

Parker, A. J. \& Newsome, W. T. 1998 Sense and the single neuron: probing the physiology of perception. A. Rev. Neurosci. 21, 227-277.

Pashler, H. E. 1998 The psychology of attention. Cambridge, MA: MIT.

Posner, M. I. 1980 Orienting of attention. Q. F. Exp. Psychol. 32, 3-25.

Reynolds, J. H., Pasternak, T. \& Desimone, R. 2000 Attention increases sensitivity of V4 neurons. Neuron 26, 703-714.

Rodman, H. R. \& Albright, T. D. 1987 Coding of visual stimulus velocity in area MT of the macaque. Vision Res. 27, 2035-2048.

Roy, J. P. \& Wurtz, R. H. 1990 The role of disparity-sensitive cortical neurons in signalling the direction of self-motion. Nature 348, 160-162.

Sade, A. \& Spitzer, H. 1998 The effects of attentional spread and attentional effort on orientation discrimination. Spat. Vision 11, 367-383.

Schaafsma, S. J. \& Duysens, J. 1996 Neurons in the ventral intraparietal area of awake monkey closely resemble neurons in the dorsal part of the medial superior temporal area in their response to optic flow. F. Neurophysiol. 76, 4056-4068.

Schaafsma, S. J., Duysens, J. \& Gielen, C. C. A. M. 1997 Responses in ventral intraparietal area of awake macaque monkey to optic flow patterns corresponding to rotation of planes in depth can be explained by translation and expansion effects. Visual Neurosci. 14, 633-646.

Sclar, G. \& Freeman, R. D. 1982 Orientation selectivity in the cat's striate cortex is invariant with stimulus contrast. Exp. Brain Res. 46, 457-461.
Skottun, B. C., Bradley, A., Sclar, G., Ohzawa, I. \& Freeman, R. D. 1987 The effects of contrast on visual orientation and spatial frequency discrimination: a comparison of single cells and behavior. F. Neurophysiol. 57, 773-786.

Snowden, R. J., Treue, S. \& Andersen, R. A. 1992 The response of neurons in area V1 and MT of the alert rhesus monkey to moving random dot patterns. Exp. Brain Res. 88, 389-400.

Spitzer, H. \& Richmond, B. J. 1991 Task difficulty: ignoring, attending to, and discriminating a visual stimulus yield progressively more activity in inferior temporal neurons. Exp. Brain Res. 83, 340-348.

Spitzer, H., Desimone, R. \& Moran, J. 1988 Increased attention enhances both behavioral and neuronal performance. Science 240, 338-340.

Tolhurst, D. J., Movshon, J. A. \& Thompson, I. D. 1981 The dependence of response amplitude and variance of cat visual cortical neurons on stimulus contrast. Exp. Brain Res. 41, 414-419.

Tolhurst, D. J., Movshon, J. A. \& Dean, A. F. 1983 The statistical reliability of signals in single neurons in cat and monkey visual cortex. Vision Res. 23, 775-785.

Treue, S. \& Martínez Trujillo, J. C. 1999 Feature-based attention influences motion processing gain in macaque visual cortex. Nature 399, 575-579.

Treue, S. \& Maunsell, J. H. R. 1999 Effects of attention on the processing of motion in macaque middle temporal and medial superior temporal visual cortical areas. $\mathcal{F}$. Neurosci. 19, 7591-7602.

Ungerleider, L. G. \& Desimone, R. 1986 Cortical connections of visual area MT in the macaque. f. Comp. Neurol. 248, 190-222.

Urbach, D. \& Spitzer, H. 1995 Attentional effort modulated by task difficulty. Vision Res. 35, 2169-2177.

Vogels, R. \& Orban, G. A. 1994 Activity of inferior temporal neurons during orientation discrimination with successively presented gratings. F. Neurophysiol. 71, 1428-1451.

Zhang, K. \& Sejnowski, T. J. 1999 Neuronal tuning: to sharpen or broaden? Neural Comput. 11, 75-84.

\section{GLOSSARY}

MST: medial superior temporal area MT: middle temporal visual area ROC: receiver operating characteristic VIP: ventral intraparietal area 